\title{
Optimum conditions for Prodigiosin production by Serratia marcescens S11
}
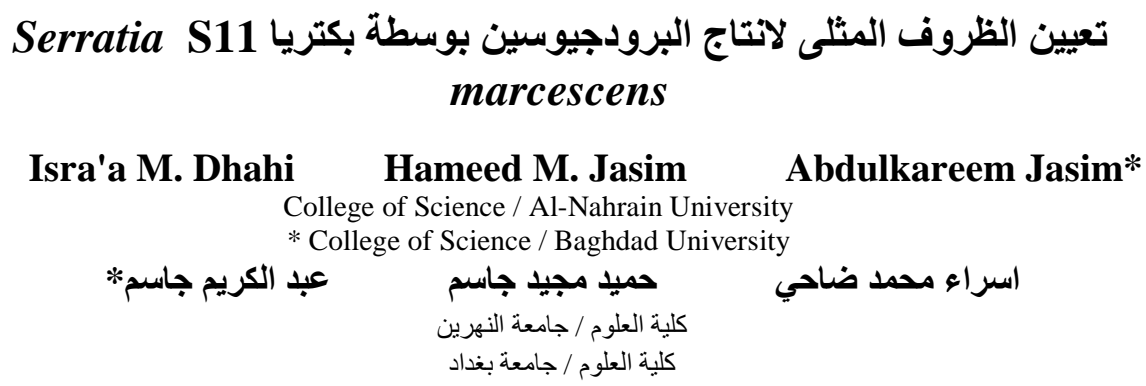

Introduction

Prodigiosin is a secondary metabolite (red pigment) produced by Serratia marcescens and many other grams negative and positive bacteria [1]. It was of great interest in medicine due to its antifungal, antibacterial, antiprotozoal, antimalarial, immunosuppressive, and anticancer activities [2, 3].

There are many conditions that affect the productivity of prodigiosin pigment, which include $\mathrm{pH}$, temperature, carbon source, nitrogen source, and phosphate source. Optimum growth of all strains of Serratia has been observed at $\mathrm{pH} 7$ and optimum $\mathrm{pH}$ for prodigiosin production is between (8.0-8.5) [4]. The temperature is considered as one of the most important factors affecting pigment productivity and the growth of the microorganisms. Optimum growth of all strains of Serratia has been observed at a temperature from (20-37) ${ }^{\circ} \mathrm{C}$, while the optimum temperature of prodigiosin production was at $30^{\circ} \mathrm{C}$ [5]. Microorganisms differ in their needs to carbon sources 
according to their nutrient nature; the use of pure carbon sources e.g. (glucose, sucrose and fructose) is expensive from the economical case, so the industrial fermentation try to use cheap carbon sources especially industrial and a variety of plant seed oils have also been used as carbon substances for prodigiosin production and displayed stimulatory effects on the production by $S$. marcescens. Mineral salts have an effect on the production of prodigiosin and there are several studies which demonstrated that synthesis of prodigiosin by non-proliferating cells of $S$. marcescens is depends on the presence of inorganic phosphate (Pi) concentrations [6,7]. According to the importance of prodigiosin in different applications, this study was aimed to determine the optimum conditions for prodigiosin production by local isolate of $S$. marcescens

\section{Materials and Methods}

S. marcescens S11 was obtained from previous study [8], and was maintained on slants of Nutrient agar medium kept at $4^{\circ} \mathrm{C}$. Fresh cultures were obtained by inoculating Nutrient broth medium with a loopful of stock culture, and incubated at 28 ${ }^{\circ} \mathrm{C}$ for 16 hours in a shaker incubator (150 rpm).

\section{Optimum conditions for prodigiosin production}

Optimization of prodigiosin production by $S$. marcescens $\mathrm{S} 11$ was carried out aerobically in the production medium (brain-heart infusion broth) under batch culture conditions. Optimum conditions include type and concentration of carbon source, type and concentration of nitrogen source, type of phosphate source, temperature, and $\mathrm{pH}$.

\section{Assay of prodigiosin activity}

Prodigiosin activity (U/cell) was measured spectrophotometrically according to Haddix [9], by inoculating brain-heart infusion broth with $1 \%$ of mid-exponential phase culture of $S$. marcescens S11 and incubated with shaking at $150 \mathrm{rpm}$ in a shaker incubator at $28^{\circ} \mathrm{C}$ until the optical density of the growth medium was reaches 0.75 at $620 \mathrm{~nm}$. At the same time, optical density of the growth medium was also measured at $499 \mathrm{~nm}$ ( $\lambda$-max of prodigiosin absorbance), then prodigiosin activity was calculated according to the following equation against blank of growth medium:

Prodigiosin activity $(\mathrm{U} / \mathrm{Cell})=$

$$
\text { [O. } \left.\mathrm{D}_{499}-\left(1.3831 \times \mathrm{O}_{620}\right)\right] \times 1000
$$

\section{Results and Discussion}

$$
\text { O. D } 620
$$

\section{Optimum conditions for prodigiosin production}

\section{Effect of Carbon Source}

Six carbon sources (fructose, glucose, sucrose, lactose, olive oil, and sunflower oil) were used as a sole source of carbon and energy to determine the optimum in prodigiosin production by $S$. marcescens $\mathrm{S} 11$, these carbon sources were added to the production medium in a concentration of $2 \%$.

The results in Figure (1) showed that the maximum production of prodigiosin was obtained when the culture medium supplemented with olive oil as a sole source for carbon and energy. The prodigiosin activity in culture medium was $440.3 \mathrm{U} /$ cell. This may due to olive oil which contains many nutrients essential for growth requirements for the microorganism such as fatty acids, growth factors, vitamins an ${ }^{1} \ldots \ldots \ldots$ 
In addition, S. marcescens has lipase activity and thereby was capable for hydrolyzing oil substrates to liberate fatty acids as a sole source for carbon and energy [11], while glucose may inhibit prodigiosin production due to catabolic repression or by lowering medium $\mathrm{pH}$ during growth and fermentation [12].

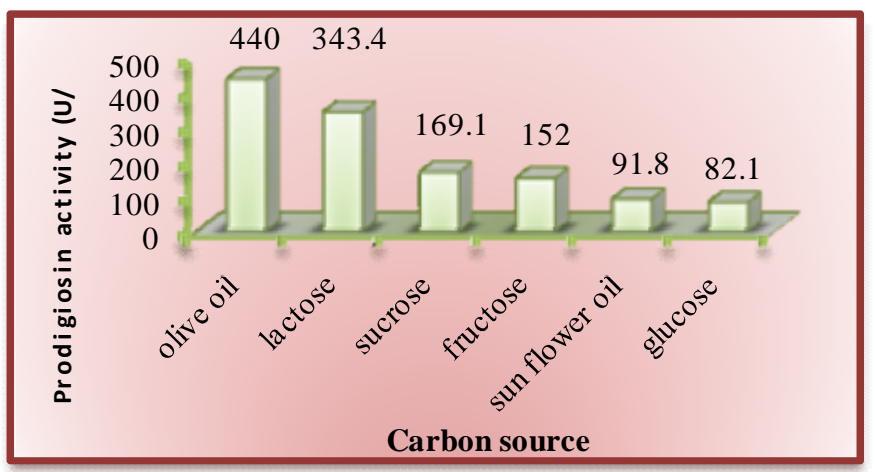

Fig (1): Effect of carbon source on prodigiosin production by $S$. marcescens S11 after incubation in shaker incubator $(150 \mathrm{rpm})$ for $24 \mathrm{hrs}$ at $28^{\circ} \mathrm{C}$.

\section{Effect of carbon source concentration}

The optimum carbon source (olive oil) was used to supplement the production medium in a concentration of $(1,1.5,2,2.5,3,3.5) \%(\mathrm{v} / \mathrm{v})$ to determine the optimum concentration for production of prodigiosin by $S$. marcescens $\mathrm{S} 11$.

Figure (2) showed that the maximum production of prodigiosin was obtained when the concentration of olive oil was $1.5 \%$, at this concentration the prodigiosin activity in culture medium was $833.3 \mathrm{U} /$ cell. This may indicate that this carbon source concentration was the best for providing the microorganism with the needed energy for growth and maximum production of the pigment. Other studies indicated that the optimum carbon and energy source for the prodigiosin production varies between different concentrations of olive oil, for example [13] referred that $4 \%$ (w/v) was the optimum carbon source for prodigiosin production by $S$. marcescens $\mathrm{SM} \Delta \mathrm{R}$.

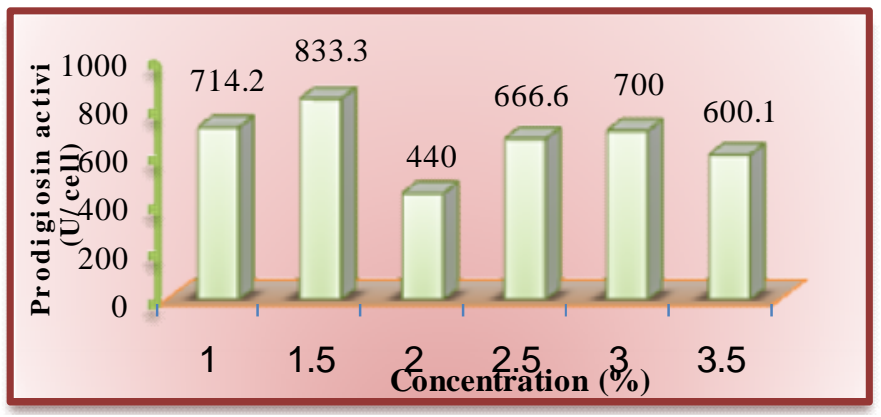

Fig (2): Effect of olive oil concentration on prodigiosin production by $S$. marcescens $\mathrm{S11}$ after incubation in shaker incubator $(150 \mathrm{rpm})$ for $24 \mathrm{hrs}$ at $28^{\circ} \mathrm{C}$.

\section{Effect of nitrogen source}

Five nitrogen sources were used to supplement the production medium for enhancing prodigiosin production by $S$. marcescens $\mathrm{S} 11$, three of these nitrogen sources are organic (peptone, tryptone, and casein hydrolysate), and two inorganic (ammonium nitrates and ammonium sulphate). These sources were added to the production medium instead of tryptone and yeast extract in a concentration of $1.5 \%$. 
The maximum production of prodigiosin was noticed in medium was supplemented with casein hydrolysate as an organic nitrogen source. The prodigiosin activity in culture medium using this nitrogen source was 922 U/cell Figure (3). This result may be attributed to the type of nitrogen source and its growth factors contents that supplements bacterial requirements for growth, production and secretion of prodigiosin to culture medium as mentioned by [13]. Furthermore, the production of prodigiosin in culture medium by $S$. marcescens 11 using organic nitrogen sources (peptone, tryptone, casein hydrolysate) was better than the prodigiosin production using inorganic nitrogen sources (ammonium sulfate, ammonium nitrate) under the same condition. The increase in the production of prodigiosin using the casein hydrolysate may be attributed to its natural component that provide the medium with nitrogen source which contributed in the supporting of bacterial biomass, also it contains trace elements such as $\mathrm{Ca}, \mathrm{Mg}$, and carbohydrates that provide the optimum condition for pigment activities especially those enzymes responsible for biosynthesis of prodigiosin, and as it was mentioned by [14].

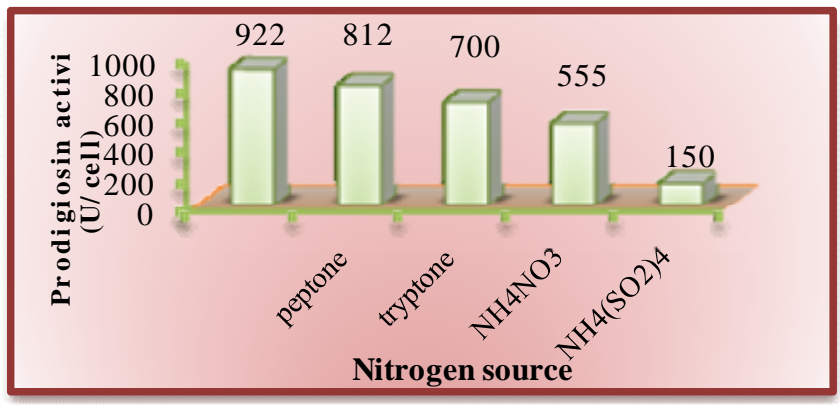

Fig (3): Effect of nitrogen source on prodigiosin production by $S$. marcescens S11 after incubation in shaker incubator $(150 \mathrm{rpm})$ for $24 \mathrm{hr}$ at $28^{\circ} \mathrm{C}$.

\section{Effect of nitrogen source concentration}

Different concentrations $(0.5,1,1.5,2,2.5) \% \mathrm{w} / \mathrm{v}$ of the optimum nitrogen source, casein hydrolysate were used to supplement the production medium.

Figure (4) showed that the maximum production of prodigiosin was recorded in concentration of $1.5 \%$ of casein hydrolysate, the prodigiosin activity was $1000 \mathrm{U} /$ cell. On the other hand, the increase or decrease of casein hydrolysate concentrations above or below the optimum concentration value causing a decrease in prodigiosin production, this may due to the change in the $\mathrm{C} / \mathrm{N}$ ratio in production medium that affects different secondary metabolites pathways especially those responsible for prodigiosin production and as it was mentioned by [13].

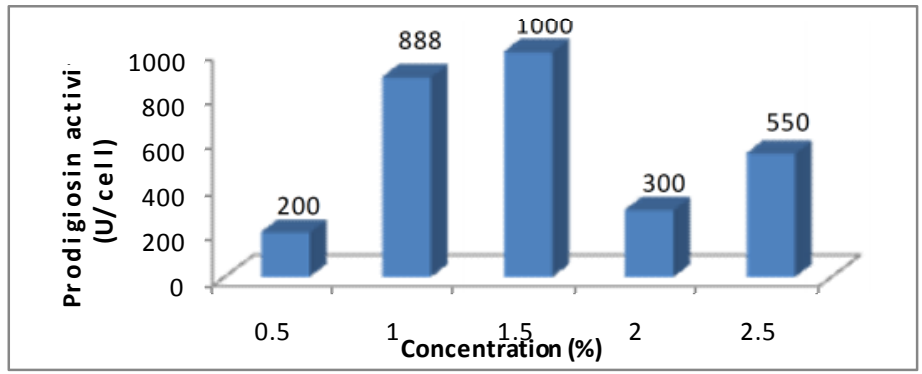

Fig (4): Effect of casein hydrolysate concentration on prodigiosin production by S. marcescens $\mathbf{S 1 1}$ after incubation in shaker incubator $(150 \mathrm{rpm})$ for 24$]$ 


\section{Effect of phosphate source}

Different phosphate sources were also studied to determine the optimum one for prodigiosin production by $S$. marcescens S11. Two types of phosphate sources $\left(\mathrm{KH}_{2} \mathrm{PO}_{4}\right.$ and $\left.\mathrm{K}_{2} \mathrm{HPO}_{4}\right)$ were added to the production medium at a concentration of $0.1 \%$, and a mixture of them $\left(0.07 \%\right.$ of $\mathrm{KH}_{2} \mathrm{PO}_{4}$ and $0.03 \%$ of $\left.\mathrm{K}_{2} \mathrm{HPO}_{4}\right)$ were also used. The results indicated that addition of $\mathrm{KH}_{2} \mathrm{PO}_{4}$ to production medium was showed an increase in prodigiosin activity $(1500 \mathrm{U} / c e l l)$ in comparison with $\mathrm{K}_{2} \mathrm{HPO}_{4}$ or when used both of them Figure (5). The presence of phosphate in culture medium works as a buffering capacity when medium become alkaline due to the biosynthesis of prodigiosin [15].

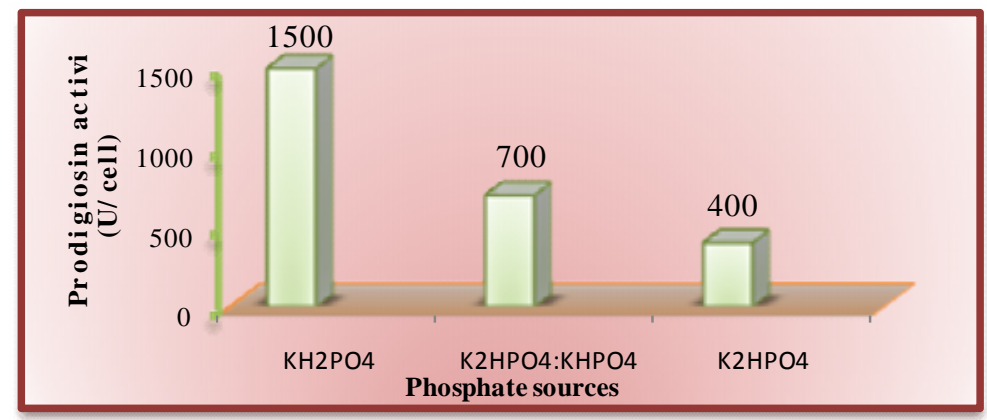

Fig (5): Effect of phosphate sources on prodigiosin production by $S$. marcescens $\mathrm{S} 11$ after incubation in shaker incubator $(150 \mathrm{rpm})$ for $24 \mathrm{hrs}$ at $28^{\circ} \mathrm{C}$.

\section{Effect of Temperature}

In order to determine the optimum incubation temperature for prodigiosin production by $S$. marcescens $\mathrm{S} 11$, different incubation temperatures $(24,28,32,36,40){ }^{\circ} \mathrm{C}$ were used for this purpose.

From Figure (6) it was found that the maximum production of prodigiosin was obtained when the temperature of fermentation medium was $28^{\circ} \mathrm{C}$. At this temperature, prodigiosin activity in culture medium was 2714 U/cell. This temperature was the optimum for bacterial metabolism and prodigiosin production as a secondary metabolite. Other studies showed that the optimum temperature for prodigiosin production was $30^{\circ} \mathrm{C}[16,17]$.

A block in prodigiosin production occurred above $30^{\circ} \mathrm{C}$ in culture medium, while the presence of fatty acids in culture medium supported prodigiosin production up to $42^{\circ} \mathrm{C}$ [8].The increase in temperature to $40^{\circ} \mathrm{C}$ led to decrease in the bacterial growth rate and made the conditions unsuitable for prodigiosin production and finally led to repress the expression of genes responsible for prodigiosin with less effect on bacterial growth (10), or may repress the genes responsible for Prodigiosin Condensing Enzyme (PCE) which are sensitive to high temperature [18]. 


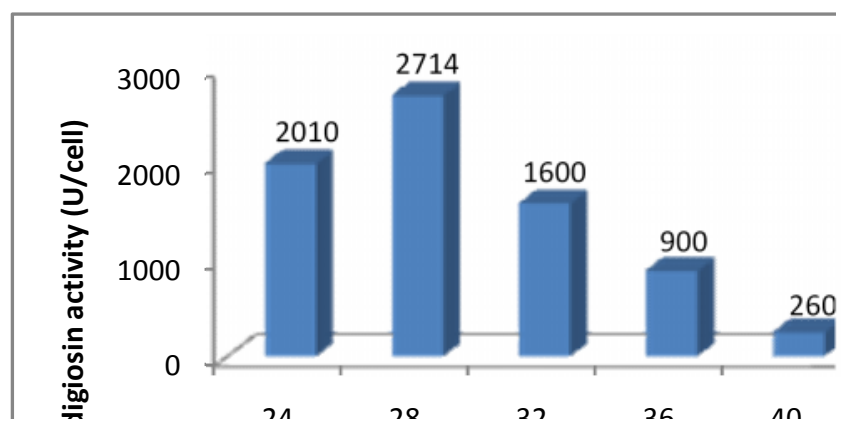

Fig (6): Effect of different incubation tempenatpereon)prodigiosin production

by $S$. marcescens $S 11$ after incubation in shaker incubator $(150 \mathrm{rpm})$ for $24 \mathrm{hrs}$ at $28^{\circ} \mathrm{C}$.

\section{Effect of $\mathbf{p H}$}

Different $\mathrm{pH}$ values $(7.0,7.5,8.0,8.5,9.0)$ were used to determine the optimum for prodigiosin production by $S$. marcescens $\mathrm{S} 11$. As it was shown in Figure (7), the maximum production of prodigiosin was obtained when the $\mathrm{pH}$ value was 8.0 , at this $\mathrm{pH}$ the prodigiosin activity in culture medium was $3000 \mathrm{U} / \mathrm{cell}$. This result was in agreement with [18] who noticed that the same result when the production medium was adjusted to alkaline $\mathrm{pH}$. In addition, results showed that the increase or decrease in the $\mathrm{pH}$ value of the production medium above or under the optimum $\mathrm{pH}$ decrease prodigiosin production, this may due to the alteration of the activities of all genes responsible for prodigiosin biosynthesis [12].

The effect of $\mathrm{pH}$ value on pigment productivity is due to two reasons, the first is the effect on the properties of the culture medium including the solubility of the nutrients, transport and ionization, and the second is the effect of $\mathrm{pH}$ on the stability of the pigment.

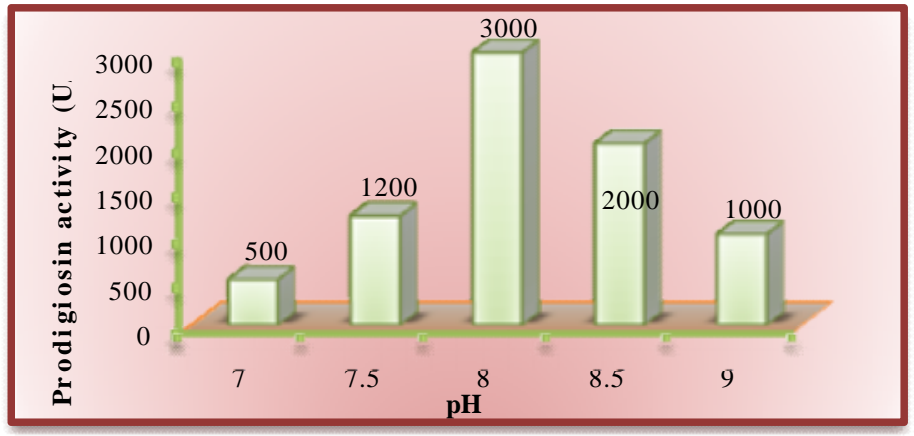

Fig (7): Effect of medium pH on prodigiosin production by $S$. marcescens $\mathrm{S11}$ after incubation in shaker incubator $(150 \mathrm{rpm})$ for $24 \mathrm{hrs}$ at $28^{\circ} \mathrm{C}$.

\section{References}

1. Singlton, P. and Sainsbury, D. (2001). Dictionary of Microbiology and Molecular Biology. 3rd Ed. Johan Willy and Sons Ltd.

2. D'Alessio, R.; Bargiotti, A.; and Carlini, O. (2000). Synthesis and immunosuppressive activity of novel prodigiosin derivatives. J. Med. Chem., 43: 2557-2565.

3. Montaner, B.; Navarro, S.; Piqué, M.; Vilaseca, M.; Martinell, M.; Giralt, E.; Gil, J. and Peréz-Tomás, R. (2000). Prodigiosin from the supernatant of Serratia marcescens induces apoptosis in haematopoietic cancer cell lines. J. Pharmacol., 131: 585-593.

4. Solem, M.; Rius, N.; Francia, A. and Loren, J. G. (1994).The effect of pH on prodigiosin production by non-proliferating cells of Serrati amarcescens. Let. in applied microbial., 19: 341-344. 
5. Holt, J. G.; Krieg, N. R.; Sneath, H. A.; Staley, J. T. and Williams, S. T. (1994). Bergy's Manual of Determinative Bacteriology. 9th Ed Williams and Wilkins. U.S.A.

6. Yamashita, M.; Nakagawa, Y.; Li, H. and Matsuyama, T. (2001). Silica gel-dependent production of prodigiosin and serrawttins by Serratia marcescens in liquid culture. Envrionmen. Microb, 16: 250-254.

7. Brivonese, A. and Sutherland, W. I. (1989). Polymer production by a mucoid strain of Azotobacter vinelandii in batch culture. App. Microbiol. Biotechnol., 30: 97-102.

8. Hameed M. J.; Isra'a M. D.; Abdulkareem J. (2010). Effect of Physical and Chemical Mutagens on Prodigiosin Production by the Locally Isolated Serratia marcescens. Unpublished data.

9. Haddix, P. L.;Paulsen, E. T.and Werner, T. F.(2000). Measurement of mutation to antibiotic resistance:Ampicillin resistance in Serratia marcescens. Bioscene: J. 26: 17-21.

10. Jama, A. (2008). Dietary alpha-linolinic acid is associated with reduced risk of fatal coronary heart disease, but increased prostate cancer risk: a meta-analysis. $J$. of Nutrition, 295:403-415.

11. Wei, Y. H. and Chen, W. C. (2005). Enhanced production of prodigiosin-like pigment from Serratia marcescens SMAR by medium improvement and oil-supplementation strategies. J. Biosci. Bioeng., 99 (6): 616-622.

12. Sole, M.; Francia, A.; Rius, N. and Lorén, J. G. (1997). The role of pH in the 'glucose effect' on prodigiosin production by non-proliferating cells of Serratia marcescens. Lett. Appl. Microbiol., 25: 81-84.

13. Kim, C. H.; Sung-Ho, K. and Suk-In, K. (1999). Isolation and Characteristics of prodigiosin like red pigment produced by Serratia sp. KH-95. Kor. J. Appl. Microbiol. Biotechnol., 26: 283-289.

14. Frank, R.; Witney, L.; and Failla, E. D. (1997). Phosphate Inhibition of Secondary Metabolism in Serratia marcescens. Americ. Society for Microbio., 33 (5): 1042-1046.

15. Robert, P.W.; Cora, L.G.; Hussain, S.M. and Randolph, H.S. (1971). Influence of temperature of incubation and type of growth medium on pigmentation in Serratia marcescens. J. Bacteriol., 106 (2): 438-443.

16. Greenwood, D.; Slack, R. C. B. and Peutherer, J. F. (2002). Med. Microbiol., 6th Ed.

17. Furstner, A. (2003). Chemistry and biology of rosephilin and the prodigiosin alkaloids: a survey of the last 2500 years. Angew. Chem. Int. Ed., 42: 3582-3603.

18. 18- Giri, A.V.; Anandkumar, N.; Muthukumaran, G. and Pennathur, G. (2004). A novel medium for the enhanced cell growth and production of prodigiosin from Serratia marcescens isolated from soil. BMC Microbiol., 4: 11. 